\title{
Differential Channel Optical Readout System for Color Changes of Gas Sensitive Colorimetric Dyes
}

\author{
C. Weber ${ }^{1,2}$, M. El-Safoury ${ }^{1}$, C. Pannek¹, L. Engel' ${ }^{1}$, A. Eberhardt ${ }^{1}$, M.-L. Bauersfeld ${ }^{1}$, J. Wöllenstein ${ }^{1,2}$ \\ ${ }^{1}$ Fraunhofer Institute for Physical Measurement Techniques IPM, Heidenhofstr. 8, 79110, Freiburg, Germany \\ ${ }^{2}$ Department of Microsystems Engineering-IMTEK, Laboratory for Gas Sensors, University of Freiburg, Georges- \\ Köhler-Allee 102, 79110, Freiburg, Germany \\ Christian.weber@ipm.fraunhofer.de
}

\begin{abstract}
Summary:
We present a simple sensor setup for detecting very small color changes of gasochromic materials. The sensor includes up to ten LEDs for capturing different spectral channels, ranging from ultraviolet to the near infrared. The System features a differential optical structure that intrinsically allows comparing the reflectance of the gas sensitive dye to that of a reference dye. This allows for detecting diminutive color changes of dyes at gas concentrations in the sub ppm range with a better signal-tonoise ratio and drift stability compared to single channel systems.
\end{abstract}

Keywords: optical readout, differential, dyes color, gas sensor, colorimetric

\begin{abstract}
Motivation
Current smoke detectors for residential use are predominantly based on the stray light principle. Detecting the light scattered by smoke particles, they feature two main disadvantages: The detection method can hardly differentiate between particles emitted from fires and harmless dust or fog particles. In addition, the detectors can only identify fires, which emit larger amounts of smoke particles. Especially for smoldering fires, this is not always the case.
\end{abstract}

In order to overcome these disadvantages, the combination with sensors for the detection of gases, emitted by fires, is advantageous [1]. The emission of carbon monoxide (CO) is a very specific indicator for burning processes and therefore, the detection of $\mathrm{CO}$ is ideally suited for this application. Measuring $\mathrm{CO}$ at an early fire stage requires a highly sensitive and selective detection method. The colorimetric gas sensing principle (also known as gasochromic principle) meets these requirements. It relies on a color changing chemical reaction of the target gas with a specifically tailored dye. In this work, we present a setup that is able to read out even very tiny color changes of gasochromic dyes in the presence carbon monoxide for fire detection purposes.

\section{Readout System for Gas Dependent Color Change}

In the presented measurement system, the color detection is accomplished by illuminating the dye with ten different LEDs and measuring the reflected light intensities in their respective spectral ranges.
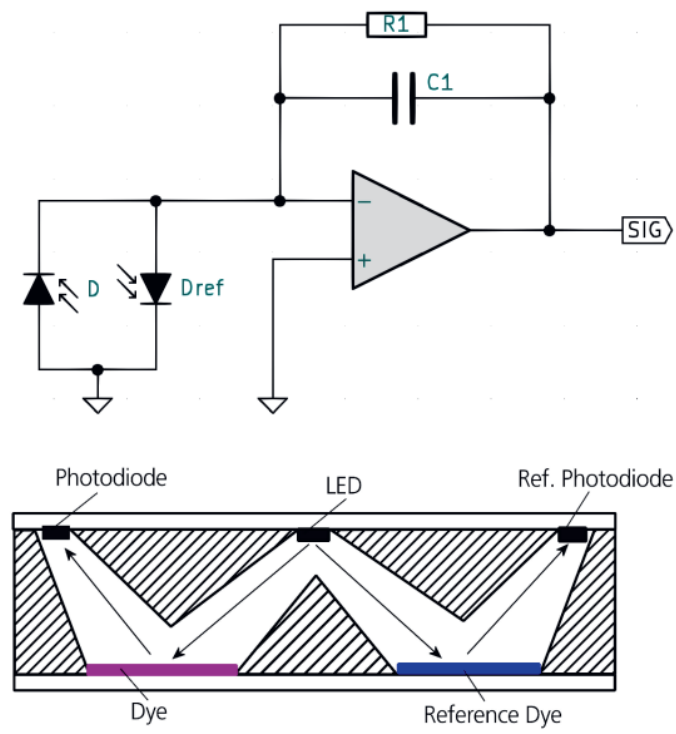

Fig. 1. Principle of the proposed optical differential circuit. The signal of the photodiode $D$ and the reference photodiode Dref are subtracted intrinsically. Together with a completely symmetric optical layout, the output signal only depends on the difference between dye and reference dye.

In a single channel measurement system, the light reaching the detector is partly also reflected by the chamber walls and carries no information of the dye color. This fraction of the light represents an unwanted offset and might even lead to sensor drift. While the offset typically 50 $80 \%$ in such a single channel signal, the color 
changes to be resolved can be as small as $10^{-3} \%$. In order to decrease the offset fraction, we propose a differential detection principle consisting of two antiparallel photodiodes with a symmetrical arrangement (see Fig. 1). The antiparallel interconnection of the photodiodes enables a intrinsic differential measurement where the offset cancels out and only the reflection difference generates a signal. For an absolute reflection measurement, there are also two single channel photodiodes placed beside the differential detectors.

As shown in Fig. 1, the system comprises a Wshaped beam path. This ensures a defined symmetrical light distribution, blocking direct light from the LEDs to the detector

The ten LEDs with wavelengths ranging from 395 to $940 \mathrm{~nm}$ are operated successively. The photodiode current signals of the single channels are amplified with $2.7 \cdot 10^{6} \mathrm{~V} / \mathrm{A}$, while the photodiode signal of the differential channel is amplified with $2.7 \cdot 10^{8} \mathrm{~V} / \mathrm{A}$. An average LED current of $900 \mu \mathrm{A}$ is modulated sinusoidal at $5 \mathrm{kHz}$, while the detector signals are captured and filtered by a digital lock-in algorithm having $1 \mathrm{~s}$ averaging time and running on an onboard PSoC6 microcontroller. Fig. 2 shows the assembled readout system

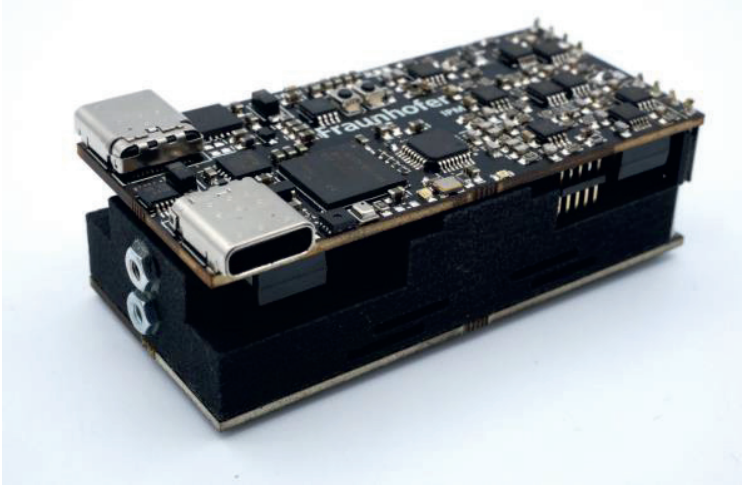

Fig. 2. Picture of the assembled system. The upper $P C B$ contains the LEDs and the detectors on the bottom side, while the dyes are located on the lower $P C B$. In between, a 3D-printed chamber assembles the $W$-shaped light path.

\section{Measurement Setup for CO}

In order to detect $\mathrm{CO}$ with the developed sensor system, it is equipped with a gasochromic dye based on a binuclear rhodium complex, which was synthesized as described in [2]. The complex reacts with $\mathrm{CO}$, showing a color change from purple to yellow. It is adsorbed on nanostructured silica particles, which were glued to PET foil and applied to the sensor system with adhesive. As reference dye, uncoated silica particles were used. The measurements with the developed setup were performed at the Fraunhofer IPM gas laboratory. The sensor system was placed in a gastight box with a volume of $500 \mathrm{~cm}^{3}$. A flow of synthetic air through the box with $50 \%$ r.h. at $2 \mathrm{l} / \mathrm{min}$ was established. By adding $\mathrm{CO}$ to the gas mixture, concentrations of 1,10 and 100 ppm CO were realized.

\section{Comparison of the Differential and the sin- gle Channel}

In Fig 3, the sensor signals of the differential channel and the single channel with the orange LED $(630 \mathrm{~nm})$ while applying different CO concentrations are depicted. In the differential channel, the color change initiated by $1 \mathrm{ppm}$ of CO can be resolved easily while the single channel hardly resolves $10 \mathrm{ppm}$. This can be attributed to the higher analog amplification that is possible in the offset free differential channel.

The differential channel is also less prone to drift introduced by the chamber optical properties (swelling, temperature change, etc).

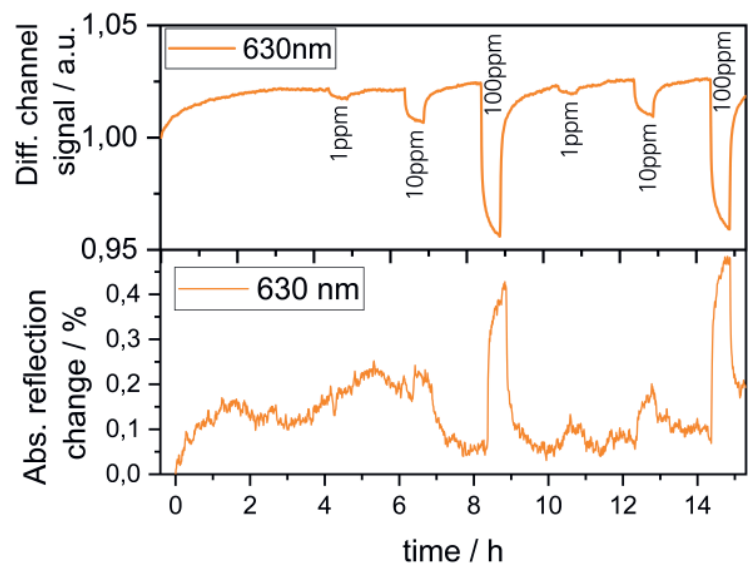

Fig. 3. Comparison of the Normalized signal of the differential detector (top) with the single channel delivering an absolute reflection change (bottom)

\section{Conclusion}

Within the scope of this work, we developed a multispectral readout circuit, which enables the detection of diminutive color changes of gasochromic dyes. Our measurement results, with a rhodium complex based dye, show the possibility to detect $1 \mathrm{ppm} \mathrm{CO}$ while showing less drift compared to a single channel measurement

\section{References}

[1] A. Duric, H. Ebner, M. Forster, I. Vinage: "Development of a multi-sensor detector for fire detection and life safety applications", 14th International Conference on Automatic Fire Detection AUBE09, Duisburg Germany 2009.

[2] F. Cotton, A. Chakravarty, D. Tocher, J. Tocher, Structural and Electrochemical Characterization of the Novel Ortho-Metalated Dirhodium(II) Compounds $\mathrm{Rh} 2(\mathrm{O} 2 \mathrm{CCH} 3) 2[(\mathrm{C} 6 \mathrm{H} 5) 2 \mathrm{P}(\mathrm{C} 6 \mathrm{H} 4) 2 * 2 \mathrm{~L}$, Organometallics 4 (1985) 8-13 